\title{
PHASE RETRIEVAL IN INFINITE-DIMENSIONAL HILBERT SPACES
}

\author{
JAMESON CAHILL, PETER G. CASAZZA, AND INGRID DAUBECHIES \\ ABSTRACT. The main result of this paper states that phase retrieval in infinite- \\ dimensional Hilbert spaces is never uniformly stable, in sharp contrast to the \\ finite-dimensional setting in which phase retrieval is always stable. This leads \\ us to derive stability results for signals depending on how well they are ap- \\ proximated by finite expansions.
}

\section{INTRODUCTION}

Given a separable Hilbert space $\mathcal{H}$, phase retrieval deals with the problem of recovering an unknown $f \in \mathcal{H}$ from a set of intensity measurements $\left(\left|\left\langle f, \varphi_{n}\right\rangle\right|\right)_{n \in I}$ for some countable collection $\Phi=\left\{\varphi_{n}\right\}_{n \in I} \subseteq \mathcal{H}$. Note that if $f=\alpha g$ with $|\alpha|=1$, then $\left|\left\langle f, \varphi_{n}\right\rangle\right|=\left|\left\langle g, \varphi_{n}\right\rangle\right|$ for every $n \in I$ regardless of our choice of $\Phi$; we say $\Phi$ does phase retrieval if the converse of this statement is true, i.e., if the equalities $\left|\left\langle f, \varphi_{n}\right\rangle\right|=\left|\left\langle g, \varphi_{n}\right\rangle\right|$ for every $n$ imply that there is a unimodular scalar $\alpha$ so that $f=\alpha g$.

We will generally assume that $\Phi$ forms a frame for $\mathcal{H}$, i.e., there are positive constants $0<A \leq B<\infty$ so that

$$
A\|f\|^{2} \leq \sum_{n \in I}\left|\left\langle f, \varphi_{n}\right\rangle\right|^{2} \leq B\|f\|^{2}
$$

for every $f$ in $\mathcal{H}$. We call the operator $T_{\Phi}: \mathcal{H} \rightarrow \ell^{2}(I)$ given by

$$
T_{\Phi}(f)=\left(\left\langle f, \varphi_{n}\right\rangle\right)_{n \in I}
$$

the analysis operator of $\Phi$. We denote by $\mathcal{A}_{\Phi}: \mathcal{H} \rightarrow \ell^{2}(I)$ the nonlinear mapping given by

$$
\mathcal{A}_{\Phi}(f)=\left(\left|\left\langle f, \varphi_{n}\right\rangle\right|\right)_{n \in I},
$$

so that $\Phi$ does phase retrieval if and only if $\mathcal{A}_{\Phi}$ is injective on $\mathcal{H} / \sim$, where $f \sim g$ if $f=\alpha g$ with $|\alpha|=1$.

Definition 1.1. We say a frame $\left\{\varphi_{n}\right\}_{n \in I}$ for a Hilbert space $\mathcal{H}$ has the complement property if for every subset $S \subseteq I$ we have

$$
\overline{\operatorname{span}}\left\{\varphi_{n}\right\}_{n \in S}=\mathcal{H} \quad \text { or } \quad \overline{\operatorname{span}}\left\{\varphi_{n}\right\}_{n \notin S}=\mathcal{H} .
$$

Received by the editors January 27, 2016 and, in revised form, June 21, 2016

2010 Mathematics Subject Classification. Primary 46C05; Secondary 94A15.

The second author was supported by NSF DMS 1609760; NSF ATD 1321779; and ARO W911NF-16-1-0008.

The third author was supported by AFOSR grant 00002113-02; ONR grant N00014-11-1-071406-7; and NSF grant DMS-1516988.

(C) 2016 by the authors under Creative Commons Attribution-Noncommercial 3.0 License (CC BY NC 3.0) 
Theorem 1.2. (a) Let $\mathcal{H}$ be a separable Hilbert space and let $\Phi$ be a frame for $\mathcal{H}$. If $\Phi$ does phase retrieval, then $\Phi$ has the complement property.

(b) Let $\mathcal{H}$ be a separable Hilbert space over the real numbers and let $\Phi$ be a frame for $\mathcal{H}$. If $\Phi$ has the complement property, then $\Phi$ does phase retrieval.

Proof. (a) Suppose $\Phi$ does not have the complement property and find $S \subseteq I$ so that neither $\left\{\varphi_{n}\right\}_{n \in S}$ nor $\left\{\varphi_{n}\right\}_{n \notin S}$ spans $\mathcal{H}$. Then we can find nonzero $f, g \in \mathcal{H}$ so that $\left\langle f, \varphi_{n}\right\rangle=0$ for all $n \in S$ and $\left\langle g, \varphi_{n}\right\rangle=0$ for all $n \notin S$. Also, since $\Phi$ is a frame we know that $f \neq \lambda g$ for any scalar $\lambda$, so in particular we know $f+g \neq 0$ and $f-g \neq 0$. It now follows that $\left|\left\langle f+g, \varphi_{n}\right\rangle\right|=\left|\left\langle f-g, \varphi_{n}\right\rangle\right|$ for all $n \in I$ but $f+g \neq \lambda(f-g)$ for any scalar $\lambda$, so $\Phi$ does not do phase retrieval.

(b) Suppose $\Phi$ does not do phase retrieval and find nonzero $f, g \in \mathcal{H}$ so that $\left|\left\langle f, \varphi_{n}\right\rangle\right|=\left|\left\langle g, \varphi_{n}\right\rangle\right|$ for every $n \in I$, but $f \neq \pm g$. Since $\mathcal{H}$ is a real Hilbert space this means that $\left\langle f, \varphi_{n}\right\rangle= \pm\left\langle g, \varphi_{n}\right\rangle$, so let $S=\left\{n \in I:\left\langle f, \varphi_{n}\right\rangle=\left\langle g, \varphi_{n}\right\rangle\right\}$. Then $f-g \neq 0$ but $\left\langle f-g, \varphi_{n}\right\rangle=0$ for every $n \in S$ so $\overline{\operatorname{span}}\left\{\varphi_{n}\right\}_{n \in S} \neq \mathcal{H}$, and similarly $f+g \neq 0$ but $\left\langle f+g, \varphi_{n}\right\rangle=0$ for every $n \notin S$ so $\overline{\operatorname{span}}\left\{\varphi_{n}\right\}_{n \notin S} \neq \mathcal{H}$, which means $\Phi$ does not have the complement property.

This theorem was originally proved in 3 where it was only stated in the finitedimensional case, but the proof still holds in infinite dimensions without any modifications. The original proof of part (a) presented in [3] did not give the correct conclusion in the case where $\mathcal{H}$ is a Hilbert space over the complex numbers. This was observed by the authors of [5] where they presented a much more complicated proof for this case. It turns out that the proof presented in [3] does hold in this case with only minor modifications, which is the proof presented above.

We remark here that recently several papers have been devoted to showing that certain frames do phase retrieval for infinite-dimensional spaces over both the real and complex numbers, so by Theorem 1.2 all of these frames have the complement property. For instance, in [10] it is shown that a real-valued band-limited signal can be recovered up to sign from the absolute values of its samples at any rate greater than twice the Nyquist rate. A similar result for complex-valued bandlimited signals was shown in [9] which required a minimal oversampling rate of four times the Nyquist rate.

In 8 the authors study an instance of the phase retrieval problem using the Cauchy wavelet transform to recover analytic functions in $L^{2}(\mathbb{R}, \mathbb{C})$ that have compactly supported Fourier transforms. In that paper they observe that although they are able to show that $\mathcal{A}_{\Phi}$ is injective (for the particular choice of $\mathcal{H}$ and $\Phi$ ) there is an inherent lack of robustness in the sense that arbitrarily small perturbations of the measurements $\mathcal{A}_{\Phi}(f)$ can result in large errors in the reconstructed signal (see sections 4.1 and 4.2 in 8 ]). The main result of the present paper states that this type of lack of robustness is unavoidable when doing phase retrieval in an infinite-dimensional Hilbert space.

In this paper, we restrict ourselves to the case of countably infinite frames in Hilbert spaces; in work extending the present results, [1 proves a similar lack of robustness for phase retrieval in infinite-dimensional Banach spaces with infinite frames that need not be countable.

One way to quantify the robustness of the phase retrieval process for a given frame $\Phi$ is in terms of the lower Lipschitz bound of the map $\mathcal{A}_{\Phi}$ with respect to some metric on the space $\mathcal{H} / \sim$. A natural choice of metric is the quotient metric 
induced by the metric on $\mathcal{H}$ given by

$$
d(\tilde{f}, \tilde{g})=\inf _{|\alpha|=1}\|f-\alpha g\| .
$$

We would like to find a positive constant $C$ (depending only on $\Phi$ ) so that for every $f, g \in \mathcal{H}$

$$
\inf _{|\alpha|=1}\|f-\alpha g\| \leq C\left\|\mathcal{A}_{\Phi}(f)-\mathcal{A}_{\Phi}(g)\right\|
$$

In [5] the authors introduced a numerical version of the complement property as a means of quantifying the constant $C$ in (1.1):

Definition 1.3. We say a frame $\left\{\varphi_{n}\right\}_{n \in I}$ has the $\sigma$-strong complement property if for every subset $S \subseteq I$ either $\left\{\varphi_{n}\right\}_{n \in S}$ or $\left\{\varphi_{n}\right\}_{n \notin S}$ is a frame for $\mathcal{H}$ with lower frame bound at least $\sigma$.

In [5] it is shown that when $\mathcal{H}=\mathbb{R}^{M}$ the lower Lipschitz bound of $\mathcal{A}_{\Phi}$ is precisely controlled by the largest $\sigma$ for which $\Phi$ has the $\sigma$-strong complement property (see also 4). Although this result does not apply to the complex case, much like the complement property cannot be used to determine whether a given frame does phase retrieval for a complex space, we still have the following result in the finitedimensional case.

Proposition 1.4. If $\mathcal{H}$ is a finite-dimensional Hilbert space and $\Phi=\left\{\varphi_{n}\right\}_{n \in I}$ does phase retrieval for $\mathcal{H}$, then $\mathcal{A}_{\Phi}$ has a positive lower Lipschitz bound, i.e., $\mathcal{A}_{\Phi}$ satisfies (1.1) for some $C<\infty$.

Proof. Since this result is already known if $\mathcal{H}$ is a real Hilbert space we will prove it for the case where $\mathcal{H}=\mathbb{C}^{N}$. Note that the inequality (1.1) is homogeneous so without loss of generality we can assume that $\|f\|=1$ and $\|g\| \leq 1$.

Let $\mathbb{H}_{N}$ denote the space of $N \times N$ Hermitian matrices equipped with the HilbertSchmidt inner product $\langle X, Y\rangle=\operatorname{Trace}(X Y)$. Because of the restriction to Hermitian matrices, this is a Hilbert space over the reals (of dimension $M^{2}$ ), and no adjoint is necessary in the definition of $\langle X, Y\rangle$. Define the linear mapping $\mathcal{A}_{\Phi}^{2}: \mathbb{H}_{N} \rightarrow \ell^{2}(I)$ by

$$
\mathcal{A}_{\Phi}^{2}(X)=\left(\left|\left\langle X, \varphi_{n} \varphi_{n}^{*}\right\rangle\right|\right)_{n \in I}=\left(\left|\left\langle X \varphi_{n}, \varphi_{n}\right\rangle\right|\right)_{n \in I},
$$

where we denote by $g g^{*}$ the rank one operator that maps $h \in \mathbb{C}^{N}$ to $\langle h, g\rangle g$. (Note that if $X$ is rank 1, i.e., $X=f f^{*}$, then $\mathcal{A}_{\Phi}^{2}(X)=\left(\left|\left\langle f, \varphi_{n}\right\rangle\right|^{2}\right)_{i \in I}$, hence the notation $\mathcal{A}_{\Phi}^{2}$.) It is well known that $\Phi$ does phase retrieval if and only if $\operatorname{ker}\left(\mathcal{A}_{\Phi}^{2}\right)$ does not contain any matrix of rank 1 or 2 (see Lemma 9 in [5]). This, together with the compactness of the set $S=\left\{X \in \mathbb{H}_{N}: \operatorname{rank}(X) \leq 2,\|X\|=1\right\}$ (since $\mathbb{H}_{N}$ is finite dimensional), implies that

$$
\min _{X \in S}\left\|\mathcal{A}_{\Phi}^{2}(X)\right\|=c>0
$$

where $\|X\|$ denotes the operator norm (however, we can choose any norm on $\mathbb{H}_{N}$ and this will still be true). 
For $f, g \in \mathbb{C}^{N}, f f^{*}-g g^{*}$ is rank 1 or 2 , so we have

$$
\begin{aligned}
\left\|f f^{*}-g g^{*}\right\|^{2} & \leq \frac{1}{c^{2}}\left\|\mathcal{A}_{\Phi}^{2}\left(f f^{*}-g g^{*}\right)\right\|^{2} \\
& =\frac{1}{c^{2}}\left\|\mathcal{A}_{\Phi}^{2}\left(f f^{*}\right)-\mathcal{A}_{\Phi}^{2}\left(g g^{*}\right)\right\|^{2} \\
& =\frac{1}{c^{2}} \sum_{n \in I}\left(\left|\left\langle f, \varphi_{n}\right\rangle\right|^{2}-\left|\left\langle g, \varphi_{n}\right\rangle\right|^{2}\right)^{2} .
\end{aligned}
$$

Furthermore, since we are assuming $\|f\|=1$ and $\|g\| \leq 1$, we have

$$
\begin{aligned}
\sum_{n \in I}\left(\left|\left\langle f, \varphi_{n}\right\rangle\right|^{2}-\left|\left\langle g, \varphi_{n}\right\rangle\right|^{2}\right)^{2} & =\sum_{n \in I}\left(\left|\left\langle f, \varphi_{n}\right\rangle\right|-\left|\left\langle g, \varphi_{n}\right\rangle\right|\right)^{2}\left(\left|\left\langle f, \varphi_{n}\right\rangle\right|+\left|\left\langle g, \varphi_{n}\right\rangle\right|\right)^{2} \\
& \leq \sum_{n \in I}\left(\left|\left\langle f, \varphi_{n}\right\rangle\right|-\left|\left\langle g, \varphi_{n}\right\rangle\right|\right)^{2}\left(2\left\|\varphi_{n}\right\|\right)^{2} \\
& \leq\left(4 \max _{n \in I}\left\|\varphi_{n}\right\|^{2}\right) \sum_{n \in I}\left(\left|\left\langle f, \varphi_{n}\right\rangle\right|-\left|\left\langle g, \varphi_{n}\right\rangle\right|\right)^{2} .
\end{aligned}
$$

Since we are assuming $\|f\| \geq\|g\|$, a direct computation shows that the largest (in absolute value) eigenvalue of $f f^{*}-g g^{*}$ is

$$
\frac{1}{2}\left(\|f\|^{2}-\|g\|^{2}+\left(\left(\|f\|^{2}+\|g\|^{2}\right)^{2}-4|\langle f, g\rangle|^{2}\right)^{1 / 2}\right) .
$$

Therefore, we have that

$$
\begin{aligned}
\left\|f f^{*}-g g^{*}\right\| & \geq \frac{1}{2}\left(\left(\|f\|^{2}+\|g\|^{2}\right)^{2}-4|\langle f, g\rangle|^{2}\right)^{1 / 2} \\
& =\frac{1}{2}\left(\|f\|^{2}+\|g\|^{2}-2|\langle f, g\rangle|\right)^{1 / 2}\left(\|f\|^{2}+\|g\|^{2}+2|\langle f, g\rangle|\right)^{1 / 2} \\
& =\frac{1}{2}\left(\inf _{|\alpha|=1}\|f-\alpha g\|\right)\left(\|f\|^{2}+\|g\|^{2}+2|\langle f, g\rangle|\right)^{1 / 2},
\end{aligned}
$$

and since $\|f\|=1$ this says

$$
\inf _{|\alpha|=1}\|f-\alpha g\| \leq 2\left\|f f^{*}-g g^{*}\right\| .
$$

Finally, combining (1.2), (1.3), and (1.4) yields (1.1).

\section{MAin RESUlts}

Before stating the main result we first remark that, when viewed as a subset of $\mathbb{C}^{M \times N}$, the set of frames $\left\{\varphi_{n}\right\}_{n=1}^{N}$ that do phase retrieval for $\mathbb{C}^{M}$ is an open subset for each $N$; see [2,7]. In fact, in [7] it is shown that if $N \geq 4 M-4$, then this set is open and dense, and it is clear that it must be empty if $N$ is sufficiently small. At this time it is not known if there exists a pair $(M, N)$ where the set of frames consisting of $N$ vectors which do phase retrieval for $\mathbb{C}^{M}$ is nonempty but not dense (see [1]), but in any case, the set of frames which do not do phase retrieval is never dense unless it is all of $\mathbb{C}^{M \times N}$. The next statement says that this situation is reversed when we consider frames for an infinite-dimensional space. 
Proposition 2.1. Let $\mathcal{H}$ be an infinite-dimensional separable Hilbert space and suppose $\left\{\varphi_{n}\right\}_{n \in \mathbb{N}}$ does phase retrieval. For every $\epsilon>0$ there is another frame $\left\{\psi_{n}\right\}_{n \in \mathbb{N}}$ which does not do phase retrieval and satisfies

$$
\sum_{n \in \mathbb{N}}\left\|\varphi_{n}-\psi_{n}\right\|^{2}<\epsilon
$$

Proof. Let $\left\{e_{n}\right\}_{n \in \mathbb{N}}$ be an orthonormal basis for $\mathcal{H}$ and choose $k \in \mathbb{N}$ so that

$$
\sum_{n=k+1}^{\infty}\left|\left\langle e_{1}, \varphi_{n}\right\rangle\right|^{2}<\epsilon .
$$

For $n \leq k$ let $\psi_{n}=\varphi_{n}$ and for $n>k$ let

$$
\psi_{n}=\sum_{i=2}^{\infty}\left\langle\varphi_{n}, e_{i}\right\rangle e_{i}
$$

Now we have that

$$
\sum_{n \in \mathbb{N}}\left\|\varphi_{n}-\psi_{n}\right\|^{2}=\sum_{n=k+1}^{\infty}\left|\left\langle e_{1}, \varphi_{n}\right\rangle\right|^{2}<\epsilon .
$$

Also, it is clear that $\left\{\psi_{n}\right\}_{n=1}^{k}$ cannot $\operatorname{span} \mathcal{H}$, and for every $n>k$ we have that $\left\langle e_{1}, \psi_{n}\right\rangle=0$, so $\left\{\psi_{n}\right\}_{n=k+1}^{\infty}$ does not span $\mathcal{H}$ either. Therefore $\left\{\psi_{n}\right\}_{n \in \mathbb{N}}$ does not have the complement property and so by Theorem 1.2 does not do phase retrieval. Furthermore, for $\epsilon$ sufficiently small $\left\{\psi_{n}\right\}_{n \in \mathbb{N}}$ is still a frame.

The above proposition suggests an infinite-dimensional space is fundamentally different from a finite-dimensional setting when doing phase retrieval. In particular, since any frame can be perturbed by an arbitrarily small amount to arrive at a frame that does not do phase retrieval, it suggests that phase retrieval for infinitedimensional spaces is inherently unstable. We now state the main result, confirming this intuition.

Theorem 2.2. Let $\mathcal{H}$ be an infinite-dimensional separable Hilbert space and let $\Phi=\left\{\varphi_{n}\right\}_{n \in \mathbb{N}}$ be a frame for $\mathcal{H}$ with frame bounds $0<A \leq B<\infty$; further suppose that $\left\|\varphi_{n}\right\| \geq c>0$ for every $n \in \mathbb{N}$. Then, for every $\delta>0$, there exist $f, g \in \mathcal{H}$ so that $\inf _{|\alpha|=1}\|f-\alpha g\| \geq 1$ but $\left\|\mathcal{A}_{\Phi}(f)-\mathcal{A}_{\Phi}(g)\right\|<\delta$.

Before proving the theorem we need a lemma.

Lemma 2.3. Let $\mathcal{H}$ be an infinite-dimensional separable Hilbert space and let $\Phi=$ $\left\{\varphi_{n}\right\}_{n \in \mathbb{N}}$ be a frame for $\mathcal{H}$ with frame bounds $0<A \leq B<\infty$. For every $\epsilon>0$ and every $N \in \mathbb{N}$ there is a $k>N$ and an $m>k$ so that

$$
\sum_{n \notin\{N+1, \ldots, m\}}\left|\left\langle\varphi_{k}, \varphi_{n}\right\rangle\right|^{2}<\epsilon
$$

Proof. Fix $\epsilon>0$ and $N \in \mathbb{N}$. Let $V=\operatorname{span}\left\{\varphi_{1}, \ldots, \varphi_{N}\right\}$ and let $P_{V}$ denote the orthogonal projection onto $V$. Let $\left\{e_{\ell}\right\}_{\ell=1}^{L}$ be an orthonormal basis for $V$ and note that

$$
\begin{aligned}
\sum_{n \in N}\left\|P_{V} \varphi_{n}\right\|^{2} & =\sum_{\ell=1}^{L} \sum_{n \in \mathbb{N}}\left|\left\langle\varphi_{n}, e_{\ell}\right\rangle\right|^{2} \\
& \leq \sum_{\ell=1}^{L} B\left\|e_{\ell}\right\|^{2}=B L
\end{aligned}
$$


So since $\sum_{n \in \mathbb{N}}\left\|P_{V} \varphi_{n}\right\|^{2}<\infty$ we can find $k>N$ so that $\left\|P_{V} \varphi_{k}\right\|^{2}<\frac{\epsilon}{2 B}$. Then

$$
\begin{aligned}
\sum_{n=1}^{N}\left|\left\langle\varphi_{k}, \varphi_{n}\right\rangle\right|^{2} & =\sum_{n=1}^{N}\left|\left\langle\varphi_{k}, P_{V} \varphi_{n}\right\rangle\right|^{2} \\
& =\sum_{n=1}^{N}\left|\left\langle P_{V} \varphi_{k}, \varphi_{n}\right\rangle\right|^{2} \\
& \leq \sum_{n \in \mathbb{N}}\left|\left\langle P_{V} \varphi_{k}, \varphi_{n}\right\rangle\right|^{2} \\
& \leq B\left\|P_{V} \varphi_{k}\right\|^{2}<B \frac{\epsilon}{2 B}=\frac{\epsilon}{2} .
\end{aligned}
$$

Now observe that

$$
\begin{aligned}
\sum_{n=k+1}^{\infty}\left|\left\langle\varphi_{k}, \varphi_{n}\right\rangle\right|^{2} & \leq \sum_{n \in \mathbb{N}}\left|\left\langle\varphi_{k}, \varphi_{n}\right\rangle\right|^{2} \\
& \leq B\left\|\varphi_{k}\right\|^{2}<\infty
\end{aligned}
$$

so there is an $m>k$ so that

$$
\sum_{n=m+1}^{\infty}\left|\left\langle\varphi_{k}, \varphi_{n}\right\rangle\right|^{2}<\frac{\epsilon}{2}
$$

Therefore

$$
\begin{aligned}
\sum_{n \notin\{N+1, \ldots, m\}}\left|\left\langle\varphi_{k}, \varphi_{n}\right\rangle\right|^{2} & =\sum_{n=1}^{N}\left|\left\langle\varphi_{k}, \varphi_{n}\right\rangle\right|^{2}+\sum_{n=m+1}^{\infty}\left|\left\langle\varphi_{k}, \varphi_{n}\right\rangle\right|^{2} \\
& <\frac{\epsilon}{2}+\frac{\epsilon}{2}=\epsilon .
\end{aligned}
$$

Note that one consequence of the above lemma is that no frame for an infinitedimensional Hilbert space can have the $\sigma$-strong complement property, regardless of how small one picks $\sigma>0$.

Proof of Theorem 2.2. We use the lemma to construct $f$ and $g$ explicitly.

Pick $\epsilon=c^{2} \delta^{2} / 4$ and $N \in \mathbb{N}$, and determine $k$ and $m$ as in the lemma, for these choices of $\epsilon, N$. Next, pick $\psi$ so that it is orthogonal to the finite-dimensional span of $\varphi_{N+1}, \ldots, \varphi_{m}$, and set $f=\varphi_{k}\left\|\varphi_{k}\right\|^{-1}+\psi$ and $g=\varphi_{k}\left\|\varphi_{k}\right\|^{-1}-\psi$.

For $n \in\{N+1, \ldots, m\}$ we have $\left\langle f, \varphi_{n}\right\rangle=\left\langle g, \varphi_{n}\right\rangle$, so that

$$
\sum_{n \in \mathbb{Z}}\left(\left|\left\langle f, \varphi_{n}\right\rangle\right|-\left|\left\langle g, \varphi_{n}\right\rangle\right|\right)^{2}=\sum_{n \notin\{N+1, \ldots, m\}}\left(\left|\left\langle f, \varphi_{n}\right\rangle\right|-\left|\left\langle g, \varphi_{n}\right\rangle\right|\right)^{2} .
$$

The triangle inequality implies that ||$z_{1}+z_{2}|-| z_{1}-z_{2}|| \leq 2\left|z_{1}\right|$ for all $z_{1}, z_{2} \in \mathbb{C}$; applying this to each term in the right-hand side of the equation, setting $z_{1}=$ $\left\langle\varphi_{k}, \varphi_{n}\right\rangle\left\|\varphi_{k}\right\|^{-1}$ and $z_{2}=\left\langle\psi, \varphi_{n}\right\rangle$, leads to

$$
\sum_{n \in \mathbb{Z}}\left(\left|\left\langle f, \varphi_{n}\right\rangle\right|-\left|\left\langle g, \varphi_{n}\right\rangle\right|\right)^{2} \leq 4 \sum_{n \notin\{N+1, \ldots, m\}}\left|\left\langle\varphi_{k}, \varphi_{n}\right\rangle\right|^{2}\left\|\varphi_{k}\right\|^{-2} \leq \frac{4 \epsilon}{c^{2}}=\delta^{2},
$$

or $\left\|\mathcal{A}_{\Phi}(f)-\mathcal{A}_{\Phi}(g)\right\| \leq \delta$. 
On the other hand, because $\psi$ and $\left\|\varphi_{k}\right\|^{-1} \varphi_{k}$ are orthogonal unit vectors, we have that, for all $\alpha \in C$ with $|\alpha|=1$,

$$
\|f-\alpha g\|^{2}=\|(1-\alpha)\| \varphi_{k}\left\|^{-1} \varphi_{k}+(1+\alpha) \psi\right\|^{2}=|1-\alpha|^{2}+|1+\alpha|^{2}=4,
$$

so that $\inf _{|\alpha|=1}\|f-\alpha g\|=2$.

Remark 2.4. Although it is not important here, it may be interesting to note that, regardless of how small $\delta$ is, the functions $f, g$ constructed in the proof lie within the closed bounded ball with radius $\sqrt{2}$ (in fact $\|f\|=\|g\|=\sqrt{2}$ ).

Since Theorem 2.2 says that we can never do phase retrieval in a robust way for an infinite-dimensional space, but Proposition 1.4 says we can basically always do it for a finite-dimensional space, it seems natural to try to use finite-dimensional approximations when working in an infinite-dimensional setting. Our next theorem is a first step in that direction; again, we first establish a lemma.

Lemma 2.5. Let $\mathcal{H}$ be a separable Hilbert space and let $\Phi$ be a frame for $\mathcal{H}$ with frame bounds $0<A \leq B<\infty$. Then for every $f, g \in \mathcal{H}$ we have

$$
\left\|\mathcal{A}_{\Phi}(f)-\mathcal{A}_{\Phi}(g)\right\| \leq B^{1 / 2} \inf _{|\alpha|=1}\|f-\alpha g\| .
$$

Proof. First note that

$$
||\left\langle f, \varphi_{n}\right\rangle|-|\left\langle g, \varphi_{n}\right\rangle|| \leq\left|\left\langle f, \varphi_{n}\right\rangle-\left\langle g, \varphi_{n}\right\rangle\right|
$$

by the reverse triangle inequality. This means that

$$
\left\|\mathcal{A}_{\Phi}(f)-\mathcal{A}_{\Phi}(g)\right\| \leq\left\|T_{\Phi} f-T_{\Phi} g\right\| \leq B^{1 / 2}\|f-g\| .
$$

Since $\mathcal{A}_{\Phi}(\alpha g)=\mathcal{A}_{\phi}(g)$ for any unimodular scalar $\alpha$, we have

$$
\left\|\mathcal{A}_{\Phi}(f)-\mathcal{A}_{\Phi}(g)\right\|=\inf _{|\alpha|=1}\left\|\mathcal{A}_{\Phi}(f)-\mathcal{A}_{\Phi}(\alpha g)\right\| \leq B^{1 / 2} \inf _{|\alpha|=1}\|f-\alpha g\| .
$$

Remark 2.6. Since Theorem 2.2 says that $\mathcal{A}_{\Phi}$ can never have a positive lower Lipschitz bound when $\Phi$ is a frame it may seem tempting to ask whether we can achieve a positive lower bound for a set that does not form a frame, i.e., a sequence $\Phi$ that does not have an upper frame bound. While this might be possible, Lemma 2.5 tells us that in this case $\mathcal{A}_{\Phi}$ cannot have a finite upper Lipschitz bound. To see this take $g=0$ in the proof of the lemma so that $\left\|\mathcal{A}_{\Phi}(f)-\mathcal{A}_{\Phi}(g)\right\|=\left\|\mathcal{A}_{\Phi}(f)\right\|=$ $\left\|T_{\Phi}(f)\right\|$ for every $f \in \mathcal{H}$. Then use the fact that $\Phi$ does not have a finite upper frame bound to produce a sequence of unit vectors $\left\{f_{n}\right\}_{n \in \mathbb{N}}$ with $\left\|T_{\Phi}\left(f_{n}\right)\right\| \rightarrow \infty$.

Theorem 2.7. Let $\mathcal{H}$ be an infinite-dimensional separable Hilbert space and let $\Phi=\left\{\varphi_{n}\right\}_{n \in \mathbb{N}}$ be a frame for $\mathcal{H}$ with frame bounds $0<A \leq B<\infty$. For each $m \in \mathbb{N}$ let $V_{m}$ be a finite-dimensional subspace of $\mathcal{H}$ so that $\operatorname{dim}\left(V_{m+1}\right)>\operatorname{dim}\left(V_{m}\right)$. Suppose there is an increasing function $G(m)$, with $\lim _{m \rightarrow \infty} G(m)=\infty$, so that the following holds for every $m$ : for every $f, g \in V_{m}$

$$
\inf _{|\alpha|=1}\|f-\alpha g\| \leq G(m)\left\|\mathcal{A}_{\Phi}(f)-\mathcal{A}_{\Phi}(g)\right\| .
$$

For $\gamma>1$ and $R>0$ define

$$
\mathcal{B}_{\gamma}(R)=\left\{f \in \mathcal{H}:\left\|f-P_{m} f\right\| \leq G(m+1)^{-\gamma} R\|f\| \text { for every } m \in \mathbb{N}\right\},
$$


where $P_{m}$ denotes the orthogonal projection onto $V_{m}$. Then for every $f, g \in \mathcal{B}_{\gamma}(R)$ we have

$$
\inf _{|\alpha|=1}\|f-\alpha g\| \leq C(\|f\|+\|g\|)^{1 / \gamma}\left\|\mathcal{A}_{\Phi}(f)-\mathcal{A}_{\Phi}(g)\right\|^{\frac{\gamma-1}{\gamma}},
$$

where $C$ depends on only $B, R, \gamma$ and $G(1)$.

Proof. Let $f, g \in \mathcal{B}_{\gamma}(R)$. We start by proving an equality of the type

$$
\inf _{|\alpha|=1}\|f-\alpha g\| \leq C^{\prime}(1+\|f\|+\|g\|)\left\|\mathcal{A}_{\Phi}(f)-\mathcal{A}_{\Phi}(g)\right\|^{\frac{\gamma-1}{\gamma}} ;
$$

equation (2.1) will then follow by an amplification trick.

If $\left\|\mathcal{A}_{\Phi}(f)-\mathcal{A}_{\Phi}(g)\right\| \geq R B^{1 / 2} G(1)^{-\gamma}$, then it follows from

$$
\|f-\alpha g\| \leq\|f\|+\|g\|
$$

that (2.2) is satisfied for $C^{\prime}=C_{1}:=\left(R^{-1} B^{-1 / 2} G(1)^{\gamma}\right)^{\frac{\gamma-1}{\gamma}}$.

Now assume that $\left\|\mathcal{A}_{\Phi}(f)-\mathcal{A}_{\Phi}(g)\right\|<R B^{1 / 2} G(1)^{-\gamma}$. Then find $m$ so that

$$
R B^{1 / 2} G(m+1)^{-\gamma} \leq\left\|\mathcal{A}_{\Phi}(f)-\mathcal{A}_{\Phi}(g)\right\| \leq R B^{1 / 2} G(m)^{-\gamma}
$$

(we can always do this since $G$ is increasing and $\lim _{m \rightarrow \infty} G(m)=\infty$ ). We have that

$$
\|f-\alpha g\| \leq\left\|f-P_{m} f\right\|+\left\|g-P_{m} g\right\|+\left\|P_{m} f-\alpha P_{m} g\right\|,
$$

and since $P_{m} f, P_{m} g \in V_{m}$ we also have that

$$
\begin{aligned}
\inf _{|\alpha|=1} & \left\|P_{m} f-\alpha P_{m} g\right\| \\
& \leq G(m)\left\|\mathcal{A}_{\Phi}\left(P_{m} f\right)-\mathcal{A}_{\Phi}\left(P_{m} g\right)\right\| \\
& \leq G(m)\left(\left\|\mathcal{A}_{\Phi}(f)-\mathcal{A}_{\Phi}\left(P_{m} f\right)\right\|+\left\|\mathcal{A}_{\Phi}(f)-\mathcal{A}_{\Phi}(g)\right\|+\left\|\mathcal{A}_{\Phi}(g)-\mathcal{A}_{\Phi}\left(P_{m} g\right)\right\|\right) \\
& \leq G(m)\left(B^{1 / 2}\left\|f-P_{m} f\right\|+\left\|\mathcal{A}_{\Phi}(f)-\mathcal{A}_{\Phi}(g)\right\|+B^{1 / 2}\left\|g-P_{m} g\right\|\right)
\end{aligned}
$$

so that

$$
\begin{aligned}
\inf _{|\alpha|=1}\|f-\alpha g\| \leq & \left(1+G(m) B^{1 / 2}\right)\left(\left\|f-P_{m} f\right\|+\left\|g-P_{m} g\right\|\right) \\
& +G(m)\left\|\mathcal{A}_{\Phi}(f)-\mathcal{A}_{\Phi}(g)\right\| .
\end{aligned}
$$

Because $f$ and $g$ are both in $\mathcal{B}_{\gamma}(R)$, we have

$$
\begin{aligned}
\left\|f-P_{m} f\right\|+\left\|g-P_{m} g\right\| & \leq G(m+1)^{-\gamma} R(\|f\|+\|g\|) \\
& \leq B^{-1 / 2}\left\|\mathcal{A}_{\Phi}(f)-\mathcal{A}_{\Phi}(g)\right\|(\|f\|+\|g\|)
\end{aligned}
$$

on the other hand, using $\left\|\mathcal{A}_{\Phi}(f)-\mathcal{A}_{\Phi}(g)\right\|<R B^{1 / 2} G(1)^{-\gamma}$ and (2.3), we derive

$$
\begin{aligned}
1+G(m) B^{1 / 2} \leq & R^{\frac{1}{\gamma}} B^{\frac{1}{2 \gamma}} G(1)^{-1}\left\|\mathcal{A}_{\Phi}(f)-\mathcal{A}_{\Phi}(g)\right\|^{-\frac{1}{\gamma}} \\
& +B^{1 / 2} R^{\frac{1}{\gamma}} B^{\frac{1}{2 \gamma}}\left\|\mathcal{A}_{\Phi}(f)-\mathcal{A}_{\Phi}(g)\right\|^{-\frac{1}{\gamma}} \\
\leq & R^{\frac{1}{\gamma}} B^{\frac{1}{2 \gamma}}\left(G(1)^{-1}+B^{1 / 2}\right)\left\|\mathcal{A}_{\Phi}(f)-\mathcal{A}_{\Phi}(g)\right\|^{-\frac{1}{\gamma}} .
\end{aligned}
$$

Finally, we also have, using (2.3) again,

$$
\begin{aligned}
G(m)\left\|\mathcal{A}_{\Phi}(f)-\mathcal{A}_{\Phi}(g)\right\| & \leq\left(\left\|\mathcal{A}_{\Phi}(f)-\mathcal{A}_{\Phi}(g)\right\|^{-1} R B^{1 / 2}\right)^{\frac{1}{\gamma}}\left\|\mathcal{A}_{\Phi}(f)-\mathcal{A}_{\Phi}(g)\right\| \\
& =R^{\frac{1}{\gamma}} B^{\frac{1}{2 \gamma}}\left\|\mathcal{A}_{\Phi}(f)-\mathcal{A}_{\Phi}(g)\right\|^{\frac{\gamma-1}{\gamma}} .
\end{aligned}
$$


Substituting all this into (2.4), we obtain

$$
\begin{aligned}
& \inf _{|\alpha|=1}\|f-\alpha g\| \\
& \quad \leq R^{\frac{1}{\gamma}} B^{\frac{1}{2 \gamma}}\left[\left(B^{-1 / 2} G(1)^{-1}+1\right)(\|f\|+\|g\|)+1\right]\left\|\mathcal{A}_{\Phi}(f)-\mathcal{A}_{\Phi}(g)\right\|^{\frac{\gamma-1}{\gamma}},
\end{aligned}
$$

which does indeed imply (2.2), with $C^{\prime}=C_{2}:=R^{\frac{1}{\gamma}} B^{\frac{1}{2 \gamma}}\left(B^{-1 / 2} G(1)^{-1}+1\right)$.

It thus follows that, for all $f, g \in \mathcal{B}_{\gamma}(R)$, (2.2) holds for $C^{\prime}=\max \left(C_{1}, C_{2}\right) ; C^{\prime}$ is completely determined by $R, B, \gamma$ and $G(1)$.

This inequality can be sharpened further by exploiting its nonhomogeneous nature. The set $\mathcal{B}_{\gamma}(R)$ is invariant under scaling: if $f \in \mathcal{B}_{\gamma}(R)$, then so are all multiples of $f$. If, given $f, g \in \mathcal{B}_{\gamma}(R)$, we write the inequality (2.2) for $f^{\prime}=M f$, $g^{\prime}=M g$, where $M \in \mathbb{R}_{+}$is to be fixed below, then we find, upon dividing both sides by $M \neq 0$,

$$
\begin{aligned}
\inf _{|\alpha|=1}\|f-\alpha g\| & \leq C^{\prime} M^{-1}[1+M(\|f\|+\|g\|)] M^{\frac{\gamma-1}{\gamma}}\left\|\mathcal{A}_{\Phi}(f)-\mathcal{A}_{\Phi}(g)\right\|^{\frac{\gamma-1}{\gamma}} \\
& =C^{\prime} M^{-\frac{1}{\gamma}}[1+M(\|f\|+\|g\|)]\left\|\mathcal{A}_{\Phi}(f)-\mathcal{A}_{\Phi}(g)\right\|^{\frac{\gamma-1}{\gamma}} .
\end{aligned}
$$

Since this inequality holds for all $M \in \mathbb{R}_{+}$, it holds in particular for the value $M=[(\gamma-1)(\|f\|+\|g\|)]^{-1}$ that minimizes the right-hand side. We obtain

$$
\inf _{|\alpha|=1}\|f-\alpha g\| \leq C^{\prime} \gamma(\gamma-1)^{\frac{1-\gamma}{\gamma}}(\|f\|+\|g\|)^{\frac{1}{\gamma}}\left\|\mathcal{A}_{\Phi}(f)-\mathcal{A}_{\Phi}(g)\right\|^{\frac{\gamma-1}{\gamma}} .
$$

Remark 2.8. We note that although (2.1) describes a type of Hölder continuity (and thus uniform continuity) for $\mathcal{A}_{\Phi}$, when restricted to $\mathcal{B}_{\gamma}(R)$, with Hölder exponent $(\gamma-1) / \gamma$, it does not establish Lipschitz continuity (which would require Hölder exponent 1). So far, most papers on the stability of phase retrieval have focused on showing Lipschitz continuity; we do not know whether Lipschitz bounds are possible within our framework, or whether these weaker Hölder-type bounds are the strongest possible here.

This theorem complements Theorem 2.2, even though uniformly stable phase retrieval is never possible in infinite-dimensional $\mathcal{H}$, Theorem 2.7 establishes that stable phase retrieval is possible for elements of $\mathcal{H}$ that can be approximated sufficiently well by finite-dimensional expansions, and quantifies the "extent" of this restricted stability.

Note that we did not require $\Phi$ to do phase retrieval for $\mathcal{H}$ in the statement of Theorem 2.7 As the following proposition shows, this is, in fact, not necessary.

Proposition 2.9. For any infinite-dimensional separable Hilbert space $\mathcal{H}$ there exists a Riesz basis $\Phi=\left\{\varphi_{n}\right\}_{n \in \mathbb{N}}$ and a sequence of subspaces $\left\{V_{m}\right\}$ so that $\left(\Phi,\left\{V_{m}\right\}\right)$ satisfies the hypotheses of Theorem 2.7.

Proof. Let $\left\{e_{n}\right\}_{n \in \mathbb{N}}$ be an orthonormal basis for $\mathcal{H}$ and for each $m \in \mathbb{N}$ let $V_{m}=$ $\operatorname{span}\left\{e_{n}\right\}_{n=1}^{m}$. For each $m$ choose $\Psi_{m}$ to be a finite set of vectors in $V_{m}$ that does phase retrieval for $V_{m}$; we know that $d_{m}:=\# V_{m} \geq r m$, where $r=2$ or $r=4$, according to whether $\mathcal{H}$ is a real or complex Hilbert space. Now number the vectors in the successive $\Psi_{m}$ consecutively, starting at $n=2$, so that $\left\{\psi_{n}\right\}_{n=D_{m-1}+2}^{D_{m}+1}=\Psi_{m}$ 
for each $m \in \mathbb{N}$, where $D_{m}=\sum_{k=1}^{m} d_{k}$ for $m \geq 1$ and $D_{0}=0$. Without loss of generality, we can normalize the vectors in each $\Psi_{m}$ such that

$$
\sum_{n=2}^{\infty}\left\|\psi_{n}\right\|^{2}<\epsilon
$$

with $\epsilon$ to be fixed below. If we let $\varphi_{n}=e_{n}+\psi_{n}$ for $n \geq 2$ and $\varphi_{1}=e_{1}$, then

$$
\sum_{n \in \mathbb{N}}\left\|\varphi_{n}-e_{n}\right\|^{2}=\sum_{n=2}^{\infty}\left\|\psi_{n}\right\|^{2}<\epsilon,
$$

so for $\epsilon$ sufficiently small $\Phi=\left\{\varphi_{n}\right\}_{n \in \mathbb{N}}$ is a Riesz basis for $\mathcal{H}$ (see, e.g., Theorem 15.3.2 in [6]).

Also, if $f \in V_{m}$, then $\left\langle f, e_{n}\right\rangle=0$ for $n \in\left\{D_{m-1}+2, \ldots, D_{m}, D_{m}+1\right\}$, since $D_{m-1}+1 \geq 1+r / 2 m(m-1) \geq m$; consequently $\left\langle f, \varphi_{n}\right\rangle=\left\langle f, \psi_{n}\right\rangle$ for $f \in V_{m}$ and $D_{m-1}+1<n \leq D_{m}+1$. This means that $\left\{\varphi_{n}\right\}_{n=D_{m-1}+2}^{D_{m}+1}$ does phase retrieval for $V_{m}$, so we can apply Proposition 1.4 to find $C_{m}<\infty$ so that

$$
\inf _{|\alpha|=1}\|f-\alpha g\| \leq C_{m}\left\|\mathcal{A}_{\Phi}(f)-\mathcal{A}_{\Phi}(g)\right\|
$$

for every $f, g \in V_{m}$. Since this can be done for each $m \in \mathbb{N}$ it follows that $\left(\Phi,\left\{V_{m}\right\}\right)$ satisfies Theorem 2.7 with $G(m)=\max _{1 \leq k \leq m} C_{k}$.

Since Riesz bases never do phase retrieval, Proposition 2.9 does indeed show that a frame in an infinite-dimensional Hilbert space need not do phase retrieval itself in order to satisfy all the conditions in Theorem 2.7. On the other hand, if $\Phi$ does phase retrieval for $\mathcal{H}$ and $\left\{V_{m}\right\}_{m \in \mathbb{N}}$ is any sequence of finite-dimensional subspaces with increasing dimensions, then it follows from Proposition 1.4 that the pair $\left(\Phi,\left\{V_{m}\right\}_{m \in \mathbb{N}}\right)$ satisfies the hypotheses of Theorem 2.7 for some function $G(m)$; see also Proposition 2.10 below.

In the formulation of Theorem 2.7, we used the infinite sequences $\mathcal{A}_{\Phi}(f):=$ $\left(\left|\left\langle f, \varphi_{n}\right\rangle\right|\right)_{n \in \mathbb{N}}$, for $f \in V_{m}$, even though this is surely overkill for elements $f$ in the finite-dimensional spaces $V_{m}$. As the following proposition shows, one can, at little cost, restrict the frame to an appropriate finitely-truncated set of vectors for each $V_{m}$ : if $\left\{\Phi,\left\{V_{m}\right\}_{m \in \mathbb{N}}\right\}$ satisfies Theorem 2.7 for the function $G(m)$, then there is an $N(m)$ for each $m$ so that $\left(\left\{\varphi_{n}\right\}_{n=1}^{N(m)},\left\{V_{m}\right\}_{m \in \mathbb{N}}\right)$ satisfies Theorem 2.7 for some function $H(m) \geq G(m)$.

Proposition 2.10. Let $\mathcal{H}$ be an infinite-dimensional separable Hilbert space and let $\left\{\varphi_{n}\right\}_{n \in \mathbb{N}}$ be a frame for $\mathcal{H}$. Let $V$ be a finite-dimensional subspace of $\mathcal{H}$ and suppose $\left\{P_{V} \varphi_{n}\right\}_{n \in \mathbb{N}}$ does phase retrieval for $V$. Then there is an $N(V)<\infty$ so that $\left\{P_{V} \varphi_{n}\right\}_{n=1}^{N(V)}$ does phase retrieval for $V$.

Proof. For notational convenience let $\psi_{n}=P_{V} \varphi_{n}$ for each $n$ and let $\Psi=\left\{\psi_{n}\right\}_{n \in \mathbb{N}}$. Also, suppose $\operatorname{dim}(V)=M$. Recall from the proof of Proposition 1.4 that $\Psi$ does phase retrieval (for $V$ ) if and only if $\operatorname{ker} \mathcal{A}_{\Psi}^{2}$ does not contain any rank 1 or 2 matrices. But ker $\mathcal{A}_{\Psi}^{2}=\operatorname{span}\left\{\psi_{n} \psi_{n}^{*}\right\}_{n \in \mathbb{N}}^{\perp}$, and $N:=\operatorname{dim}\left(\operatorname{span}\left\{\psi_{n} \psi_{n}^{*}\right\}_{n \in \mathbb{N}}\right) \leq$ $\operatorname{dim}\left(\mathbb{H}_{M}\right)=M^{2}$. Therefore there exists a subset $I \subset \mathbb{N}$ with $|I|=N$ and $\operatorname{span}\left\{\psi_{n} \psi_{n}^{*}\right\}_{n \in I}=\operatorname{span}\left\{\psi_{n} \psi_{n}^{*}\right\}_{n \in \mathbb{N}}$, which means ker $\mathcal{A}_{\Psi_{I}}^{2}=\operatorname{ker} \mathcal{A}_{\Psi}^{2}$ and so $\left\{\psi_{n}\right\}_{n \in I}$ does phase retrieval for $V$. 
Note that in the requirement that $\left\{P_{V} \varphi_{n}\right\}_{n \in \mathbb{N}}$ does phase retrieval for $V$, i.e., up to global phase, any $f \in V$ can be reconstructed from the sequence $\left(\left|\left\langle f, P_{V} \varphi_{n}\right\rangle\right|\right)_{n \in \mathbb{N}}$, one can equally well drop the projector $P_{V}$, since $\left\langle f, P_{V} \varphi_{n}\right\rangle=\left\langle f, \varphi_{n}\right\rangle$.

The frame used in the proof of Proposition 2.9 was rather contrived, and would not be used in any concrete applications. It is reasonable to wonder how the function $G$ of Theorem 2.7 behaves for choices of $\Phi$ and $\left\{V_{m}\right\}$ of practical interest. It turns out that it may grow very fast, as illustrated by the following example.

Example 2.11. Consider the space $\mathcal{H}$ of real, square integrable functions with bandlimit $\pi$, i.e., with Fourier transform supported on $[-\pi, \pi]$, and the functions $\varphi_{n} \in \mathcal{H}$ defined, for $n \in \mathbb{Z}$, by

$$
\varphi_{n}(x)= \begin{cases}\frac{\sin \left(\pi\left(x-\frac{n}{4}\right)\right)}{\pi\left(x-\frac{n}{4}\right)} & \text { if } x \neq \frac{n}{4} \\ 1 & \text { if } x=\frac{n}{4} .\end{cases}
$$

Note that the $\varphi_{4 \ell}, \ell \in \mathbb{Z}$, constitute the standard "Shannon" orthonormal basis of $\mathcal{H}$. As shown in [10], $\Phi:=\left\{\varphi_{n}\right\}_{n \in \mathbb{Z}}$ does phase retrieval for $\mathcal{H}$.

Define the spaces $V_{n} \subset \mathcal{H}$ as the $(2 n+1)$-dimensional subspaces of $\mathcal{H}$ spanned by the $\varphi_{4 \ell},-n \leq \ell \leq n$. Then there exist functions $f_{m}, g_{m}$ in $V_{2 m}$ with sufficiently fast inverse polynomial decay such that, for some $m$-independent constant $C>0$, the following inequality holds for all $m \in \mathbb{N}$ :

$$
\inf _{\alpha \in\{1,-1\}}\left\|f_{m}-\alpha g_{m}\right\|>C(m+1)^{-1} 2^{3 m}\left\|\mathcal{A}_{\Phi}(f)-\mathcal{A}_{\Phi}(g)\right\| .
$$

Proof. We construct $f_{m}$ and $g_{m}$ explicitly.

We start by defining, for $k \in \mathbb{N}$, the functions $s_{k} \in \mathcal{H}$ recursively by setting $s_{0}(x)=\varphi_{0}(x)$ and, for $k>0, s_{k}(x):=s_{k-1}(x+1)+s_{k-1}(x)$; equivalently $s_{k}(x)=\sum_{\ell=0}^{k}\left(\begin{array}{l}k \\ l\end{array}\right) \varphi_{0}(x+\ell)$. By induction on $k$, one easily checks that, for $x \notin\{0,-1, \ldots,-k\}$,

$$
s_{k}(x)=\frac{1}{\pi} \frac{k ! \sin \pi x}{x(x+1) \ldots(x+k)} ;
$$

for $x \in\{0,-1, \ldots,-k\}$, i.e., $x=-n$ with $n \in\{0,1, \ldots, k\}$, one has $s_{k}(-n)=\left(\begin{array}{l}k \\ n\end{array}\right)$.

Next, we define

$$
f_{m}(x):=s_{m}(x+m)+s_{m}(x-2 m) \text { and } g_{m}(x):=s_{m}(x+m)-s_{m}(x-2 m) ;
$$

equivalently, in a more symmetric form that makes clear that $f_{m}, g_{m}$ are both in $V_{2 m}$,

$$
\begin{aligned}
f_{m}(\cdot) & =\sum_{\ell=0}^{m}\left(\begin{array}{c}
m \\
\ell
\end{array}\right)\left[\varphi_{0}(\cdot+2 m-\ell)+\varphi_{0}(\cdot-2 m+\ell)\right] \\
& =\sum_{\ell=0}^{m}\left(\begin{array}{c}
m \\
\ell
\end{array}\right)\left[\varphi_{4(-2 m+\ell)}+\varphi_{4(2 m-\ell)}\right] \\
g_{m}(\cdot) & =\sum_{\ell=0}^{m}\left(\begin{array}{c}
m \\
\ell
\end{array}\right)\left[\varphi_{0}(\cdot+2 m-\ell)-\varphi_{0}(\cdot-2 m+\ell)\right] \\
& =\sum_{\ell=0}^{m}\left(\begin{array}{c}
m \\
\ell
\end{array}\right)\left[\varphi_{4(-2 m+\ell)}-\varphi_{4(2 m-\ell)}\right] .
\end{aligned}
$$


Then

$$
\inf _{\alpha \in\{1,-1\}}\left\|f_{m}-\alpha g_{m}\right\|^{2}=4 \min \left(\left\|s_{m}(\cdot-2 m)\right\|^{2},\left\|s_{m}(\cdot+m)\right\|^{2}\right)=4 \sum_{\ell=0}^{m}\left[\left(\begin{array}{c}
m \\
\ell
\end{array}\right)\right]^{2} .
$$

Combining the identity $\sum_{\ell=0}^{m}\left(\begin{array}{c}m \\ \ell\end{array}\right)=2^{m}$ with

$$
\sum_{\ell=0}^{m}\left(a_{\ell}\right)^{2} \leq\left[\sum_{\ell=0}^{m} a_{\ell}\right]^{2} \leq(m+1) \sum_{\ell=0}^{m}\left(a_{\ell}\right)^{2},
$$

valid for all nonnegative numbers $a_{0}, \ldots, a_{m}$, and making use of the orthonormality of the $\varphi_{4 n}$ in $\mathcal{H}$, we thus have

$$
\inf _{\alpha \in\{1,-1\}}\left\|f_{m}-\alpha g_{m}\right\|^{2} \geq 4(m+1)^{-1} 2^{2 m} .
$$

We now estimate $\left\|\mathcal{A}_{\Phi}\left(f_{m}\right)-\mathcal{A}_{\Phi}\left(g_{m}\right)\right\|^{2}$. Since, for all $h \in \mathcal{H},\left\langle h, \varphi_{\ell}\right\rangle=h(\ell / 4)$, we have

$$
\begin{aligned}
\left\|\mathcal{A}_{\Phi}\left(f_{m}\right)-\mathcal{A}_{\Phi}\left(g_{m}\right)\right\|^{2} & \\
= & \sum_{n \in \mathbb{Z}}\left(\left|f_{m}(n / 4)\right|-\left|g_{m}(n / 4)\right|\right)^{2} \\
= & \sum_{k \in \mathbb{Z}}\left[\left(\left|f_{m}(k)\right|-\left|g_{m}(k)\right|\right)^{2}+\left(\left|f_{m}\left(k+\frac{1}{4}\right)\right|-\left|g_{m}\left(k+\frac{1}{4}\right)\right|\right)^{2}\right. \\
& \left.\quad+\left(\left|f_{m}\left(k+\frac{1}{2}\right)\right|-\left|g_{m}\left(k+\frac{1}{2}\right)\right|\right)^{2}+\left(\left|f_{m}\left(k-\frac{1}{4}\right)\right|-\left|g_{m}\left(k-\frac{1}{4}\right)\right|\right)^{2}\right] \\
=: & T_{0}+T_{1}+T_{2}+T_{-1} .
\end{aligned}
$$

Since $s_{m}(k+m)=0$ unless $k \in\{-m,-m-1, \ldots,-2 m\}$ and $s_{m}(k-2 m)=0$ unless $k \in\{2 m, 2 m-1, \ldots, m\}$, we have $\left|f_{m}(k)\right|=\left|g_{m}(k)\right|$ for all $k \in \mathbb{Z}$, so that $T_{0}=0$.

To estimate the other three terms $T_{1}, T_{2}$ and $T_{-1}$, we first observe that, for $a, b \in \mathbb{R}$

$$
(|a+b|-|a-b|)^{2}=4 \min (|a|,|b|)^{2} .
$$

Using the explicit formula (2.6) for $s_{k}(x)$, we can also rewrite $f_{m}$ and $g_{m}$ as

$$
\begin{aligned}
f_{m}(x)=\frac{m ! \sin \pi x}{\pi}[ & \frac{(-1)^{m}}{(x+m)(x+m+1) \ldots(x+2 m)} \\
& \left.+\frac{1}{(x-m)(x-m-1) \ldots(x-2 m)}\right], \\
g_{m}(x)=\frac{m ! \sin \pi x}{\pi} & {\left[\frac{(-1)^{m}}{(x+m)(x+m+1) \ldots(x+2 m)}\right.} \\
& \left.-\frac{1}{(x-m)(x-m-1) \ldots(x-2 m)}\right] .
\end{aligned}
$$

Combining these two observations, we find that

$$
\left(\left|f_{m}(x)\right|-\left|g_{m}(x)\right|\right)^{2}=\frac{4(m !)^{2}(\sin \pi x)^{2}}{\pi^{2} \prod_{s=m}^{2 m}(|x|+s)^{2}},
$$


and thus

$$
\begin{aligned}
\| \mathcal{A}_{\Phi}( & \left.f_{m}\right)-\mathcal{A}_{\Phi}\left(g_{m}\right) \|^{2} \\
& =\frac{4(m !)^{2}}{\pi^{2}} \sum_{k \in \mathbb{Z}}\left[\frac{1}{\prod_{s=m}^{2 m}(|k+1 / 2|+s)^{2}}+\frac{1}{\prod_{s=m}^{2 m}(|k+1 / 4|+s)^{2}}\right] \\
& \leq \frac{8(m !)^{2}}{\pi^{2}} \sum_{k=0}^{\infty}\left[\frac{1}{\prod_{s=m}^{2 m}(k+1 / 2+s)^{2}}+\frac{1}{\prod_{s=m}^{2 m}(k+1 / 4+s)^{2}}\right] \\
& \leq \frac{16(m !)^{2}}{\pi^{2}} \sum_{k=0}^{\infty} \frac{16(m !)^{2}}{\prod^{2}} \sum_{k=0}^{\infty} \frac{[m !]^{2}}{(m+k)^{2}[(2 m) !]^{2}} \\
& \leq \frac{16}{\pi^{2}}\left[\left(\begin{array}{c}
2 m \\
m
\end{array}\right)\right]^{-2} \sum_{k=0}^{\infty} \frac{1}{(1+k)^{2}} \leq \frac{32}{\pi^{2}}\left[\left(\begin{array}{c}
2 m \\
m
\end{array}\right)\right]^{-2} .
\end{aligned}
$$

By Stirling's formula, $\left(\begin{array}{c}2 m \\ m\end{array}\right) \sim S m^{-1 / 2} 2^{2 m}$ as $m \rightarrow \infty$, for some $m$-independent $S>0$, so that, for sufficiently large $m$,

$$
\left\|\mathcal{A}_{\Phi}\left(f_{m}\right)-\mathcal{A}_{\Phi}\left(g_{m}\right)\right\|^{2} \leq \frac{36 m}{\pi^{2} S^{2}} 2^{-4 m},
$$

and consequently, combining this with (2.7),

$$
\frac{\inf _{\alpha \in\{1,-1\}}\left\|f_{m}-\alpha g_{m}\right\|}{\left\|\mathcal{A}_{\Phi}\left(f_{m}\right)-\mathcal{A}_{\Phi}\left(g_{m}\right)\right\|} \geq \frac{\pi S \cdot 2^{3 m}}{3(m+1)} .
$$

Note that since the frame $\Phi$ in this example does phase retrieval for $\mathcal{H}$ (see [10]), it must do phase retrieval for each $V_{m} \subset \mathcal{H}$, meaning that (by the argument used in the proof of Proposition 2.10) there must indeed exist $N(m)$ and $H(m)$ such that, for all $f, g \in V_{m}$

$$
\begin{aligned}
\inf _{|\alpha|=1}\|f-\alpha g\| & \leq H(m)\left[\sum_{n=1}^{N(m)}\left(\left|\left\langle f, \varphi_{n}\right\rangle\right|-\left|\left\langle g, \varphi_{n}\right\rangle\right|\right)^{2}\right]^{1 / 2} \\
& \leq H(m)\left\|\mathcal{A}_{\Phi}(f)-\mathcal{A}_{\Phi}(g)\right\|,
\end{aligned}
$$

i.e., (2.1) must be satisfied for $G(m)=\max _{1 \leq k \leq m} H(k)$. The computation above tells us that, no matter how large we pick the $N(m), G$ must grow at least as fast as indicated by (2.5).

\section{ACKNowledgments}

The authors wish to thank the reviewer for a careful reading of the paper, pointing out several inaccuracies, and suggesting a shortcut for the proof of Theorem 2.2 .

\section{REFERENCES}

[1] R. Alaifari and P. Grohs, Phase retrieval in the general setting of continuous frames for Banach spaces, arXiv preprint (2016) arXiv:1604.03163v1.

[2] R. Balan, Stability of phase retrievable frames, SPIE Optical Engineering + Applications, International Society for Optics and Photonics, 2013; DOI : 10.1117/12.2026135. 
[3] Radu Balan, Pete Casazza, and Dan Edidin, On signal reconstruction without phase, Appl. Comput. Harmon. Anal. 20 (2006), no. 3, 345-356, DOI 10.1016/j.acha.2005.07.001. MR2224902

[4] Radu Balan and Yang Wang, Invertibility and robustness of phaseless reconstruction, Appl. Comput. Harmon. Anal. 38 (2015), no. 3, 469-488, DOI 10.1016/j.acha.2014.07.003. MR.3323113

[5] Afonso S. Bandeira, Jameson Cahill, Dustin G. Mixon, and Aaron A. Nelson, Saving phase: injectivity and stability for phase retrieval, Appl. Comput. Harmon. Anal. 37 (2014), no. 1, 106-125, DOI 10.1016/j.acha.2013.10.002. MR3202304

[6] Ole Christensen, An introduction to frames and Riesz bases, 2nd ed., Applied and Numerical Harmonic Analysis, Birkhäuser/Springer, [Cham], 2016. MR3495345

[7] Aldo Conca, Dan Edidin, Milena Hering, and Cynthia Vinzant, An algebraic characterization of injectivity in phase retrieval, Appl. Comput. Harmon. Anal. 38 (2015), no. 2, 346-356, DOI 10.1016/j.acha.2014.06.005. MR.3303679

[8] Stéphane Mallat and Irène Waldspurger, Phase retrieval for the Cauchy wavelet transform, J. Fourier Anal. Appl. 21 (2015), no. 6, 1251-1309, DOI 10.1007/s00041-015-9403-4. MR.3421917

[9] Volker Pohl, Fanny Yang, and Holger Boche, Phaseless signal recovery in infinite dimensional spaces using structured modulations, J. Fourier Anal. Appl. 20 (2014), no. 6, 1212-1233, DOI 10.1007/s00041-014-9352-3. MR.3278866

[10] Gaurav Thakur, Reconstruction of bandlimited functions from unsigned samples, J. Fourier Anal. Appl. 17 (2011), no. 4, 720-732, DOI 10.1007/s00041-010-9144-3. MR2819174

[11] C. Vinzant, A small frame and a certificate of its injectivity, arXiv preprint (2015) arXiv: 1502.04656.

Department of Mathematical Sciences, New Mexico State University, Las Cruces, NEW MEXICO 88003

Department of Mathematics, University of Missouri, Columbia, Missouri 65211

Department of Mathematics, Duke University, Durham, North Carolina 27708 